\title{
Magnetic field generation in relativistic $\operatorname{shocks}\left({ }^{*}\right)$
}

\author{
J. Wiersma and A. AchterberG \\ Sterrekundig Instituut Utrecht, University of Utrecht - The Netherlands
}

(ricevuto il 23 Maggio 2005; pubblicato online il 22 Settembre 2005)

\begin{abstract}
Summary. - Linear theory of the Weibel instability cannot explain magnetic field generation in relativistic shock fronts in electron-proton plasmas. The fireball model for Gamma-ray Burst afterglows requires a magnetic field in similar shock fronts between the fireball and the surrounding matter to explain the detected nonthermal afterglow radiation. We consider an analytical model of pre-shock protons penetrating the hot post-shock electron plasma. The linear Weibel instability produces magnetic fields through self-enhancing current channels. Perturbations with a length-scale comparable to the electron skin depth reach the highest magnetic field before the linear theory breaks down. The electrons quench the linear proton instability so that it cannot randomize the proton velocity distribution and only converts a small fraction of the available kinetic energy of the protons into magnetic fields. We conclude that the linear Weibel instability that dominates in pair plasmas is relatively unimportant in electron-proton plasmas and that non-linear processes are probably much more important.
\end{abstract}

PACS 52.27.Ny - Relativistic plasmas.

PACS 52.35.Qz - Micro-instabilities.

PACS 52.35.Tc - Shock waves and discontinuities.

PACS 98.70.Rz - $\gamma$-ray sources; $\gamma$-ray bursts.

PACS 01.30.Cc - Conference proceedings.

\section{1. - Introduction}

In relativistic shock fronts, plasma instabilities convert a significant fraction of the bulk kinetic energy into magnetic energy (e.g., $[11,4,3])$. Linear theory can describe the processes responsible for exponential magnetic field growth in electron-positron plasmas $[11,7]$ but when both electrons and heavier particles are present [4], linear theory fails. This paper discusses an analytical investigation of the linear phase and shows where this difference comes from. A more detailed discussion can be found in [10].

$\left(^{*}\right)$ Paper presented at the "4th Workshop on Gamma-Ray Burst in the Afterglow Era", Rome, October 18-22, 2004.

(C) Società Italiana di Fisica 
The fireball model explains the afterglows of Gamma-ray Bursts as radiation coming from the external shocks that form when a relativistically expanding fireball (or jet) interacts with surrounding gas [8]. These shocks probably run through an electron-proton plasma like the interstellar medium or the wind of the Gamma-ray Burst progenitor. The model can only account for the non-thermal spectrum of the afterglow radiation if the relativistic shock fronts that produce the radiation contain near-equipartition magnetic fields (e.g., [5]), and detailed modeling requires knowledge of the magnetic field configuration.

\section{2. - Weibel-like instabilities}

The mixing of pre- and post-shock plasma in a collisionless shock front produces a plasma with an anisotropic velocity distribution. Such interpenetrating beams are unstable to spontaneously growing transverse waves [9] and self-exciting longitudinal oscillations. In the case of a relativistic shock (moving almost with the speed of light) the transverse waves grow faster than the longitudinal oscillations [2].

These transverse waves involve advection currents (proportional to the beam velocity) that arise from charge bunching in the beams. These will spontaneously grow in strength because charges moving in parallel attract each other. The linear theory models these advection currents with plane waves that grow in strength resembling current channels with a fixed mutual distance for a particular wave mode. This mechanism is often referred to as the Weibel instability. Non-linear effects such as merging or bending of the current channels or interaction of different wave modes are not included in this linear theory.

\section{3. - Analysis}

To estimate what happens in electron-proton plasmas we will adapt analytical calculations done previously for an electron-positron plasma [11]. We consider pre-shock protons penetrating the post-shock electron plasma. This describes a region behind the shock front where the small-scale electromagnetic fields have already randomized the electron velocity distribution but not the proton distribution.

We obtain the simplest expressions when we assume that the proton plasma has no thermal velocity spread and that there are two identical counterstreaming beams. This approximates the configuration that arises in numerical simulations (for example, fig. 6 of [4]). The momentum distribution $F(\vec{p})$ for the protons is then

$$
F(\vec{p})=\frac{n_{\mathrm{p}}}{2}\left(\delta\left(p_{x}-p_{\mathrm{b}}\right)+\delta\left(p_{x}+p_{\mathrm{b}}\right)\right) \delta\left(p_{y}\right) \delta\left(p_{z}\right)
$$

where $n_{\mathrm{p}}$ is the total number density of the protons and $p_{\mathrm{b}}$ is the initial momentum of the protons. We will assume that the electrons have completed the shock transition so that they form a hot background plasma with thermal energy density [1]

$$
e_{\mathrm{e}}=\gamma_{\mathrm{b} 0} n_{\mathrm{e}} m_{\mathrm{e}} c^{2}
$$

where $\gamma_{\mathrm{b} 0}=\left(1+p_{\mathrm{b}}^{2} /\left(m_{\mathrm{p}} c\right)^{2}\right)^{1 / 2}$ and $n_{\mathrm{e}}$ is the electron density. Here we assume that we can neglect the pre-shock magnetic field and the thermal energy density compared to the rest mass energy density.

A plane wave perturbation with wave vector in the shock plane will grow exponentially according to linear theory. The linear theory will break down when the perturbations 
become too strong. We will estimate the magnetic field at that point by using eq. (24) from Yang et al. [11], who interpret this as the point where the magnetic field is so strong that it traps the particles that take part in the instability:

$$
B_{\text {trap }}=\frac{\gamma_{\mathrm{b} 0} m_{\mathrm{p}} c}{q v_{\mathrm{b}}} \frac{\sigma^{2}}{k}
$$

where $v_{\mathrm{b}}=p_{\mathrm{b}} /\left(\gamma_{\mathrm{b} 0} m_{\mathrm{p}}\right)$ is the initial velocity of the protons, $\sigma$ is the growth rate of the instability and $k$ is the wave number of the plane wave perturbation.

The growth rate of the instability satisfies a dispersion relation that depends on the distribution function of the involved particles. For an electron-proton plasma with properties given by eqs. (1) and (2) the dispersion relation is (see eq. (20) of [10] with $\left.v_{z 0}=0\right)$ :

$$
\sigma^{2}=\frac{k^{2} \hat{\omega}_{\mathrm{pp}}^{2} v_{\mathrm{b}}^{2}}{k^{2} c^{2}+\tilde{\omega}_{\mathrm{pe}}^{2}}
$$

where the plasma frequencies are

$$
\hat{\omega}_{\mathrm{pp}}^{2} \equiv \frac{\omega_{\mathrm{pp}}^{2}}{\gamma_{\mathrm{b} 0}}=\frac{4 \pi q^{2} n_{\mathrm{p}}}{\gamma_{\mathrm{b} 0} m_{\mathrm{p}}}, \quad \tilde{\omega}_{\mathrm{pe}}^{2}=\frac{4 \pi q^{2} n_{\mathrm{e}}}{m_{\mathrm{e}} h},
$$

where $h=\left(e_{\mathrm{e}}+P_{\mathrm{e}}\right) /\left(n_{\mathrm{e}} m_{\mathrm{e}} c^{2}\right) \simeq 4 \gamma_{\mathrm{b} 0} / 3$ is the enthalpy per unit rest mass energy. The derivation of eq. (4) assumes that $\hat{\omega}_{\text {pp }} \ll \tilde{\omega}_{\text {pe }}$.

Combining eqs. (3) and (4), we find that the mode with wave number $k=\tilde{\omega}_{\text {pe }} / c$ can reach the highest magnetic field $B_{\text {peak }}$ with

$$
B_{\text {peak }}=\frac{\gamma_{\mathrm{b} 0} m_{\mathrm{p}} v_{\mathrm{b}} \hat{\omega}_{\mathrm{pp}}^{2}}{2 q \tilde{\omega}_{\mathrm{pe}}}
$$

We can assume that $n_{\mathrm{p}} \simeq n_{\mathrm{e}}$ to ensure charge neutrality so that $\hat{\omega}_{\mathrm{pp}}^{2} \simeq\left(4 m_{\mathrm{e}} / 3 m_{\mathrm{p}}\right) \tilde{\omega}_{\mathrm{pe}}^{2}$ and using $v_{\mathrm{b}} \simeq c$ for ultra-relativistic shocks we find

$$
B_{\text {peak }} \simeq \frac{2 \gamma_{\mathrm{b} 0} m_{\mathrm{e}} c \tilde{\omega}_{\mathrm{pe}}}{3 q}
$$

As a measure of the strength of this magnetic field we can compare its energy density $e_{B}=B_{\text {peak }}^{2} /(8 \pi)$ to the initial energy density of the protons $e_{\mathrm{p}}=\gamma_{\mathrm{b} 0} n_{\mathrm{p}} m_{\mathrm{p}} c^{2}$ :

$$
\epsilon_{B} \equiv \frac{e_{B}}{e_{\mathrm{p}}} \simeq \frac{m_{\mathrm{e}}}{6 m_{\mathrm{p}}} \sim 10^{-4}
$$

\section{4. - Discussion}

The low value of $\epsilon_{B}$ in eq. (8) indicates that the linear Weibel instability can only convert a small fraction of the bulk kinetic energy of the protons into magnetic fields. In fact, the proton instability in a background of electrons cannot generate much more magnetic energy than the electron instability produces. This is a result of the response of the electrons to the proton instability, which produces the $\tilde{\omega}_{\mathrm{pe}}^{2}$ term in the denominator 
of expression (4) for the growth rate of the instability. Only the properties of the electron plasma determine the maximum magnetic field strength (7) at the end of the linear phase.

Numerical simulations of electron-ion shocks [4] do show magnetic field generation beyond the value of $\epsilon_{B}$ in eq. (8) accompanied by a growth of the length scale of the magnetic field (merging of the current channels). The details of the responsible process are still unclear, although Medvedev et al. [6] have discussed a similar process as a mechanism for suppressing the diffusion of the small-scale magnetic fields after the magnetic field generation has stopped.

The linear Weibel instability is very fast: the growth rate is of the order of the plasma frequency and this explains why the magnetic field strength can grow exponentially to a maximum value in electron-positron shocks (e.g., fig. 5 in [11]). That this process does not work for proton beams in an electron background might explain why the magnetic field grows much more gradual in simulations of electron-ion shocks (fig. 4 in [4]).

\section{5. - Conclusions}

Our analytical investigation of the linear Weibel instability of proton beams in a background plasma of hot electrons shows that the electrons have a quenching effect on the proton instability. The linear instability breaks down well before it has utilized all the bulk kinetic energy of the proton beams, and it cannot be responsible for the randomization of the proton momentum distribution.

In view of numerical results it is likely that other processes than the linear Weibel instability will generate near-equipartition magnetic fields. So rather than a thin layer of exponentially growing magnetic fields, there might be a larger region of (for example) coalescing electric current channels behind the shock front.

To extend the analysis presented above we can determine the spectrum of length scales of the magnetic fields at the end of the linear phase. Based on this we can further investigate the possible (non-)linear processes that might take over magnetic field generation. If we have a better handle on these things we will be able to predict the radiation spectrum of Gamma-ray Burst afterglows more accurately.

This research is supported by the Netherlands Research School for Astronomy (NOVA).

\section{REFERENCES}

[1] Blandford R. D. and McKee C. F., Phys. Fluids, 19 (1976) 1130-1138.

[2] Califano F., Cecchi T. and Chiuderi C., Phys. Plasmas, 9 (2002) 451-457.

[3] Fonseca R. A., Silva L. O., Tonge J. W., Mori W. B. and Dawson J. M., Phys. Plasmas, 10 (2003) 1979-1984.

[4] Trier Frederiksen J., Hededal C. B., Haugbølle T. and Nordlund A., ApJ, 608 (2004) L13-L16.

[5] Gruzinov A. and Waxman E., ApJ, 511 (1999) 852-861.

[6] Medvedev M. V., Fiore M., Fonseca R. A., Silva L. O. and Mori W. B., ApJ, 618 (2005) L75-L78.

[7] Medvedev M. V. and Loeb A., ApJ, 526 (1999) 697-706.

[8] Mészáros P. and Rees M. J., ApJ, 476 (1997) 232-237.

[9] Weibel E. S., Phys. Rev. Lett., 2 (1959) 83-84.

[10] Wiersma J. and Achterberg A., A 6 A, 428 (2004) 365-371.

[11] Yang T.-Y. B., Arons J. and Langdon A. B., Phys. Plasmas, 1 (1994) 3059-3077. 